\title{
Behavioral Rating Scale
}

National Cancer Institute

\section{Source}

National Cancer Institute. Behavioral Rating Scale. NCI Thesaurus. Code C81235.

A range of values designed to quantify the qualitative responses of an individual to a set of challenges. 